\title{
Developmentof Image Enhancement and the Feature Extraction Techniques on Rural Fingerprint Images to Improve the Recognition and the Authentication Rate.
}

\author{
Babasaheb V. Bhalerao, Ramesh R. Manza. \\ Department of Computer Science and Information Technology, \\ Dr. Babasaheb Ambedkar Marathwada University, Aurangabad (MS) India.
}

\begin{abstract}
Fingerprint recognition is one of the most popular and successful methods used for person identification which takes advantage of the fact that the fingerprint has some unique characteristics called minutiae which are points where a extracts the ridges and bifurcation from a fingerprint image. A critical step in studying the statistics of fingerprint minutiae is to reliably extract minutiae from the fingerprint images. However fingerprint images are rarely of perfect quality. Fingerprint image enhancement techniques are employed prior to minutiae extraction to obtain a more reliable estimation of minutiae locations.

Fingerprint matching is often affected by the presence of intrinsically low quality fingerprints and various distortions introduced during the acquisition process. In this paper we have used the rural fingerprints database which is collected from IIIT Delhi research lab which consists of 1634 fingerprints images. Out of which we have preprocess 600 sample preprocessing extracts the ridges and bifurcation from a fingerprint image and tried to improve the quality of images. The Resultant images quality is verified by using different quality measures.
\end{abstract}

Keywords: minutiae extraction, extracts the ridges and bifurcation, rural fingerprint authentication.

\section{Introduction}

Fingerprint matching is among the most widely used biometric technologies with a broad range of both government and civilian applications such as a UIDAI, passport control, ATM/credit card, The key challenge in fingerprint matching is getting a match decision between a pair of fingerprints from the same finger under various within-class variations. Our implementation mainly noisy rural fingerprint images enhancement, image segmentation, minutiae extraction.

Fingerprint recognition or fingerprint authentication refers to the automated method of verifying a match between two human fingerprints. Fingerprints are one of many forms of biometrics used to identify an individual and verify their identity. Because of their uniqueness and consistency over time, fingerprints have been used for over a century.

The word "Biometrics" comes from the Greek language and is derived from the words "Biomeans life and „metric" means to be measure, so biometrics is a field of science and technology used to be measure life characteristics. Biometrics System uses physical and behavioral parameters for person identification. Biometric data are unique for each individual person, even two identical twins. Basically we can use physical parameters than behavioral because behavioral parameters are changed with age and environment whereas physical parameters never changed during whole life. Fingerprint matching techniques are divided into three main types:

*Correlation based matching

* Minutiae based matching

* Pattern based matching.

Minutiae based matching is the most popular and most widely used technique for fingerprint matching. This technique refers the analysis of some unique point's exhibit on fingerprint called minutiae points. The detection and representation of these points are also known as minutiae set. There are two basic minutiae points are majorly used for matching in minutiae based technique, that is Ridge-end, which means the end of the ridges and Bifurcation points, which means one single ridge divided into two ridges.

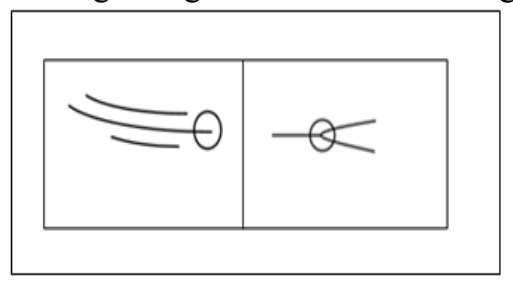

Figure 1.Ridges-end point, Bifurcation point 


\section{Fingerprint Recognition:}

Fingerprint recognition system can be used to analyze two fingerprint images one is original image and another one is template image stored in the database. Fingerprint recognition is mainly divided in two sub-parts: one is verification system and other is identification system. Fingerprint verification is used to verify the authenticity of one person with one to one matching of the database, while fingerprint identification is used to specify the person identification with one to $\mathrm{n}$ matching, fingerprint verification is rapid execution method than fingerprint identification. Fingerprint identification is especially serviceable for criminal investigation cases.

MATLAB software provides the best image processing toolbox. Digital fingerprint images can analyze easily using MATLAB. In this paper we present the result of implementation of algorithm on MATLAB.

\section{Methodology}

The complete algorithm is as follows: Input: Introduce fingerprint Image. Output: Matching score or total number of both ridges-end and bifurcation points.

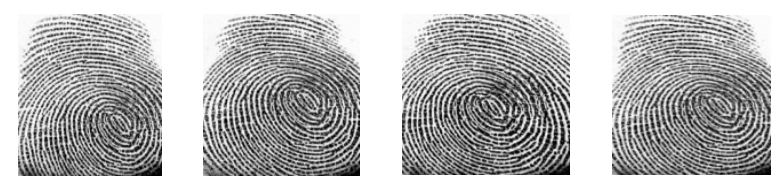

Figure2.Rural Fingerprint Original Images

Step1: Acquisition of fingerprint image. Step2: Convert image into binary form.

Step3: Apply thinning process on the binary image.

Step4: Find total numbers of ridges-end points and bifurcation points.

Step5: Match both minutiae points for fingerprint verification.

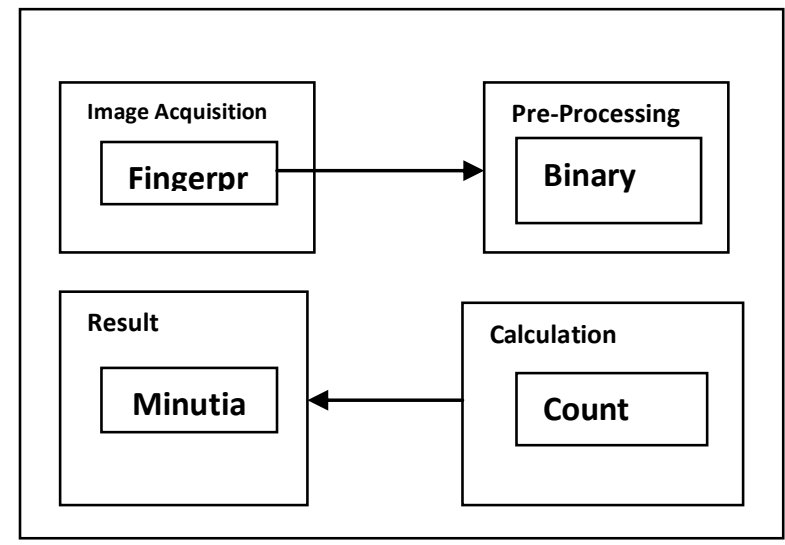

Figure 3.Flow

\section{Pre-Processing:}

Pre-processing is the process of alter a gray scale image to a black and white image. In MATLAB, a value of one represents that the pixel is white and value of zero represents that the pixel is black. This modification of gray scale image to binary image is executed by using threshold process to the image. When a threshold process is applied to an image, each pixel values are analyzed to the input threshold. Those pixel values which are smaller than the threshold value is place to zero and those pixel value which are greater than the threshold value is place to one. At the end of this process each pixel values within the image are either zero or one, and the image has been modifying to binary form. After this conversion the ridges in the fingerprint are highlighted with black color while valleys are highlighted with white color.

\section{Minutiae Extraction:}

In fingerprint extraction have an accurate representation of the fingerprint image is critical to automatic fingerprint identification systems, because most deployed commercial\&non commercial large-scale systems are dependent on feature-based matching Among all the fingerprint features, minutia point features with corresponding orientation maps are unique enough to differenceamongst fingerprints robustly; the minutiae feature representation reduces the complex fingerprint recognition problem to a point pattern matching problem. In order to achieve high-accuracy minutiae with varied quality fingerprint images, segmentation algorithm needs to separate foreground from noisy background which includes all ridge-valley regions and not the background. 
Image enhancement andFeature Extraction Techniques algorithm needs to keep the original ridge flow pattern without altering the singularity, join broken ridges, clean artifacts between pseudo-parallel ridges, and not introduce false information. Finally minutiae detection algorithm needs to locate efficiently and accurately the minutiae points

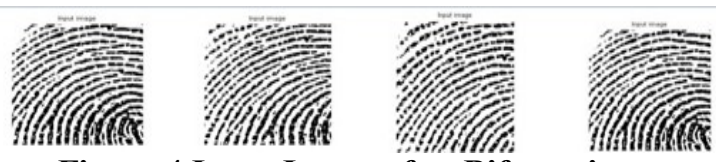

Figure 4.Input Image after Bifurcations

Thinning:

After binarization, next leading pre-processing technique used for matching process is thinning. Image thinning is the process ofdecrease thethickness of all ridges lines intosingle pixelwidth. Thinning process does not convert the original $\mathrm{x}, \mathrm{y}$ location and angle of direction of the minutiae points of the image, which assure the true calculation of minutiae points. It is also known as Block Filtering. Ridges thinning are used to destruct the extra pixel of ridges till the ridges are just one pixel broad this is done using MATLAB.
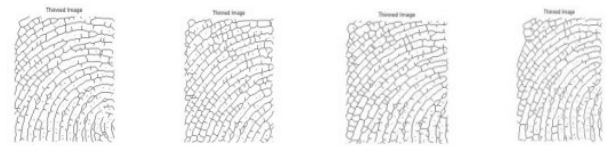

Figure 5Input images after thinning Minutiae

Input images after thinningMinutiae extraction:

Rural Fingerprint analysis using minutiae extraction process with the combination of several techniques for image pre-processing and post-processing steps to improve the input image until it is suitable for minutiae extraction. Preprocessing steps are crucial for further minutiae extraction. The Minutiae Extraction is done by

\section{Points Searching:}

The proposed algorithm detects the Minutiae Points on the basis of Ridges are disconnected at arbitrary point: Ridge-end Point and Ridges are associated with bifurcations: Bifurcation Point.

The classification of ridge-end or bifurcation points is done in MATLAB by creating matrix. If the central pixel is one, has only one neighbor pixel that is ridge-end point. Whereas, if the central pixel is one, has two neighbor pixel that is bifurcation point.

\section{Minutiae Matching:}

Image Acquisition

Fingerprint image. $\mathrm{BMP}=$ Input Image acquisition from reader.

Timage $\mathrm{BMP}=$ Template Image retrieve from database.

\section{Computation of Value:}

This step is a very important part of fingerprint matching. After the detection of minutiae points, matching algorithm require to calculate total number of available points in the fingerprint image separately. To perform this computation two counter variables are used to count both ridge-end and bifurcation points.

\section{Minutiae extraction Bifurcations value}

Table 1: Minutiae extraction Bifurcations value

\begin{tabular}{|c|c|c|}
\hline Sr.No & Name of Fingerprint Image & $\begin{array}{l}\text { Bifurcation } \\
\text { (Value) }\end{array}$ \\
\hline 1 & BB $\mid$ rural fingerprint $\mid 01 \_16 m p$ & 522 \\
\hline 2 & BB|rural fingerprint $101 \_2 \mathrm{bmp}$ & 699 \\
\hline 3 & $\overline{B B} \backslash$ rural fingerprint $\backslash 01 \_3 \mathrm{bmp}$ & 578 \\
\hline 4 & BB|rural fingerprint 01 46mp & 701 \\
\hline 5 & BB $\mid$ rural fingerprint $101 \_5 \mathrm{bmp}$ & 380 \\
\hline 6 & BB \rural fingerprint $\mid 01 \_6 b m p$ & 488 \\
\hline 7 & BB $\mid$ rural fingerprint $101 \_7 \mathrm{bmp}$ & 623 \\
\hline 8 & $\mathrm{BB} \mid$ rural fingerprint $\mathrm{l01} \mathbf{8} \mathrm{bmp}$ & 690 \\
\hline 9 & BB $\backslash$ rural fingerprint $101 \_9 \mathrm{bmp}$ & 362 \\
\hline
\end{tabular}




\begin{tabular}{|c|c|c|}
\hline 10 & BB|rural fingerprint $101 \_10 \mathrm{bmp}$ & 296 \\
\hline 11 & BB|rural fingerprint/2_1bmp & 830 \\
\hline 12 & $\overline{B B} \mid$ rural fingerprint $\mid 2 \_2 b m p$ & 760 \\
\hline 13 & BB $\mid$ rural fingerprint $\mid 2 \_3 \mathrm{bmp}$ & 857 \\
\hline 14 & BB/rural fingerprint/2_4bmp & 773 \\
\hline 15 & $\overline{B B} \mid$ rural fingerprint $\mid 2 \_5 b m p$ & 811 \\
\hline 16 & BB/rural fingerprint $\mid 2$ _6 $6 \mathrm{mp}$ & 651 \\
\hline 17 & BB $\backslash$ rural fingerprint $\mid 2 \_7 \mathrm{bmp}$ & 664 \\
\hline 18 & BB \rural fingerprint $\mid 2 \_8 b m p$ & 752 \\
\hline 19 & BB $\backslash$ rural fingerprint $\mid 2 \_9 \mathrm{bmp}$ & 675 \\
\hline 20 & $\mathrm{BB} \backslash$ rural fingerprint 12 10bmp & 775 \\
\hline 21 & BB \rural fingerprint $\mid 3$ 3_1bmp & 788 \\
\hline 22 & $\mathrm{BB} \backslash$ rural fingerprint $\mid 3 \_2 \mathrm{bmp}$ & 685 \\
\hline 23 & BB $\backslash$ rural fingerprint $\mid 3 \_3 \mathrm{bmp}$ & 786 \\
\hline 24 & $\overline{B B} \backslash$ rural fingerprint $\mid 3 \_4 \mathrm{bmp}$ & 942 \\
\hline 25 & BB $\backslash$ rural fingerprint 3 3_5bmp & 957 \\
\hline 26 & BB $\backslash$ rural fingerprint $13 \_6 \mathrm{bmp}$ & 823 \\
\hline 27 & BB \rural fingerprint $\mid 3 \_7 \mathrm{bmp}$ & 709 \\
\hline 28 & BB $\mid$ rural fingerprint $\mid 3 \_8 \mathrm{bmp}$ & 515 \\
\hline 29 & 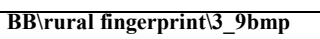 & 645 \\
\hline 30 & $\mathrm{BB} \backslash$ rural fingerprint 13 10bmp & 579 \\
\hline 31 & BB $\mid$ rural fingerprint $\mid 4$ _1bmp & 1224 \\
\hline 32 & BB $\mid$ rural fingerprint $44 \_2 \mathrm{bmp}$ & 488 \\
\hline 33 & $\mathrm{BB} \backslash$ rural fingerprint $\mid 4 \_3 \mathrm{bmp}$ & 794 \\
\hline 34 & BB \rural fingerprint $\backslash 4 \_4 \mathrm{bmp}$ & 745 \\
\hline 35 & BB $\backslash$ rural fingerprint $\mid 4 \_5 \mathrm{bmp}$ & 507 \\
\hline 36 & BB $\backslash$ rural fingerprint $\mid 4 \_6 \mathrm{bmp}$ & 690 \\
\hline 37 & $\overline{B B} \backslash$ rural fingerprint $\backslash 4 \_7 \mathrm{bmp}$ & 696 \\
\hline 38 & BB $\backslash$ rural fingerprint $\mid 4 \_8 \mathrm{bmp}$ & 420 \\
\hline 39 & BB $\mid$ rural fingerprint 44 9bmp & 672 \\
\hline 40 & BB \rural fingerprint $\backslash 4 \_10 \mathrm{bmp}$ & 594 \\
\hline
\end{tabular}

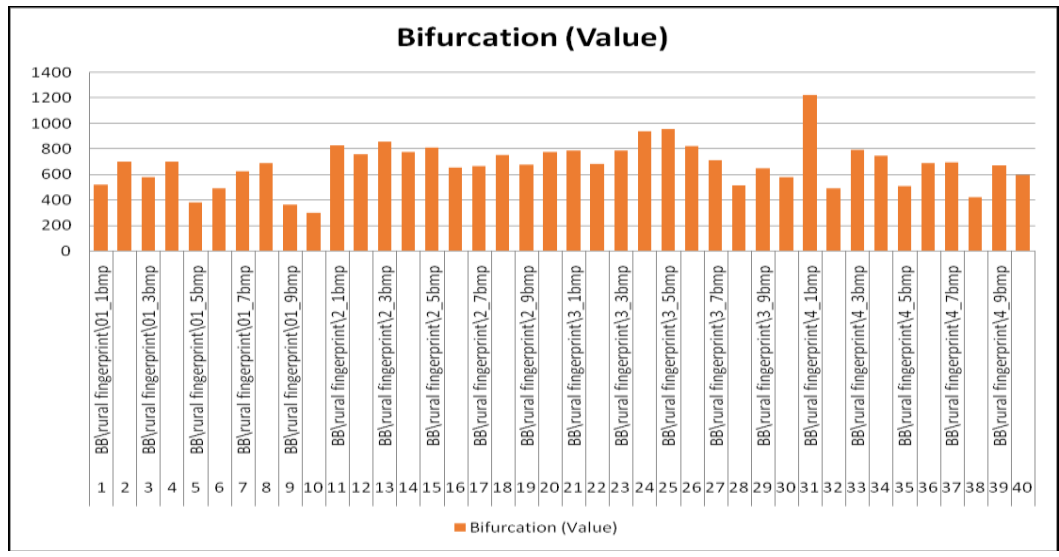

Fig 6 Bifurcations valueGraph

Each minutiae point in the fingerprint image has a specific location. This location information of particular point is significant to store for further matching of fingerprints. The location of every point in the digital image is given by pixel position, so that it can be taken and stored separately for both ridge-end and bifurcation points.
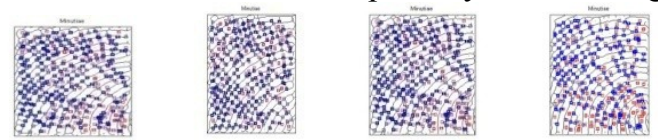

\section{Figure 7Minutiae point extracted in input images}

\section{Conclusion:}

In large scale deployment of fingerprint recognition systems, especially in rural fingerprints database, there are some challenges involved. Along with the sensor noise and poor image quality, presence of scars and 
warts, and deteriorating ridge/minutiae patterns in fingerprints from rural population affect the data distribution. Since there is no research that evaluates the performance of automatic fingerprint verification/identification in rural fingerprints databases. We will studied the performance using standard fingerprint recognition systems and fingerprint databases collected from the rural fingerprints databases, Indian population now a day's it has been work on unique identification where but when there is enhancement with such noisy fingerprints hence our research work concentrated on same. In this paper we have perform minutiae extraction fingerprint images of rural fingerprint image database. We have selected 600 images out of 1632. In this paper sample result of first forty images out of six hundred given.Fingerprint image enhancement techniques are employed prior to minutiae extraction to obtain a more reliable estimation of minutiae locations very much useful to enhance the quality of rural fingerprint image database.

\section{Acknowledgement:}

We are very much thankful to Image Analysis and Biometrics (IAB) Lab at Indraprastha Institute of Information Technology, IIIT Delhi for providing rural fingerprint database\& Special thanks SAP DSR Phase-I of Dept of Computer Science \&IT, Dr. Babasaheb Ambedkar Marthwada University, and Aurangabad.

\section{References}

[1] BabasahebV. Bhalerao, Ramesh R. ManzaYogesh M. Rajput Department of CSand IT, Dr.BabasahebAmbedkar Marthwada University, Aurangabad.(MS), India" Use of Quality Measures for Rural Indian Fingerprint Image Database Enhancement and Improve the Recognition Rate International Journal of Computer Applications (0975- 8887) Volume 70- No.18, May 2013.

[2] M. Vatsa, R. Singh, S. Bharadwaj, H. Bhatt, R. Mashruwala “Analyzing Fingerprints of Indian Population Using Image Quality": A UIDAI Case Study 1 - IIIT Delhi, India 2 - UIDAI, India.

[3] C. Puri, K. Narang, A. Tiwari, M. Vatsa, and R. Singh, “On Analysis of Rural and Urban Indian Fingerprint Images” IIIT Delhi, India.

[4] D. Maltoni, D. Maio, A. Jain, and S. Prabhakar, “Hand-book of fingerprint recognition”. Springer Verlag, 2003.

[5] R. M.Mandi,S. S. Lokhande, Rotation "Invariant Fingerprint Identification System "International Journal of Electronics Communication and Computer Technology (IJECCT) Volume 2 Issue 4 (July 2012).

[6] Rohit Singh (Y6400), Utkarsh Shah (Y6510), Vinay Gupta (Y6534), "Fingerprint Recognition”, Department of Computer Science $\&$ engineering Indian Institute of technology, Kanpur. Computer Vision and Image. Processing (CS676).

[7] Graig T. Diefenderfer,"Fingerprint Recognition "Naval PostgraduateSchool Monterey, California.

[8] ChiragDadlani, Arun Kumar Passi ,Herman SahotaMitinKrishan Kumar, "Fingerprint recognition using minutiae based feature" As part of EE851: Biometrics.

[9] Mary Lourde R, and DushyantKhosla "Fingerprint Identification in Biometric Security Systems" International Journal of Computer and Electrical Engineering, Vol. 2, No. 5, October, 2010 1793-8163.

[10] D.Maio and D. Maltoni. Direct gray-scale minutiae detection in fingerprints.IEEE Trans. Pattern Anal. And Machine Intell., 19(1):27-40, 1997.

[11] L. Hong, Y. Wan and A.K. Jain, "Fingerprint Image Enhancement: Algorithms andPerformance Evaluation", IEEE Transactions on PAMI, Vol. 20, No. 8, pp.777-789, August 1998.

[12] D. Maltoni and D. Maio, "Handbook of Fingerprint Recognition.3 Fingerprint Analysis and Representation", Springer Verlag, ISBN 0-387-95431-7, 2003

[13] T. Jea and V. Govindaraju, "A Minutia-Based Partial Fingerprint Recognition System", Pattern Recognition 2005.

[14] "Digital Image processing using MATLAB" by Rafael C.Gonzalez, Richard E.Woods, Steven L.Eddins". 